Voix et Images

voixetimages

\title{
Entretien avec Élise Turcotte
}

Denise Brassard

Volume 31, numéro 3 (93), printemps 2006

Élise Turcotte

URI : https://id.erudit.org/iderudit/013235ar

DOI : https://doi.org/10.7202/013235ar

Aller au sommaire du numéro

Éditeur(s)

Université du Québec à Montréal

\section{ISSN}

0318-9201 (imprimé)

1705-933X (numérique)

Découvrir la revue

Citer ce document

Brassard, D. (2006). Entretien avec Élise Turcotte. Voix et Images, 31(3), 15-30. https://doi.org/10.7202/013235ar d'utilisation que vous pouvez consulter en ligne.

https://apropos.erudit.org/fr/usagers/politique-dutilisation/ 


\title{
ENTRETIEN AVEC ÉLISE TURCOTTE
}

\author{
$+++$ \\ DENISE BRASSARD \\ Université du Québec à Montréal
}

voIX ET IMAGES Élise Turcotte, votre écriture entretient un lien étroit avec l'enfance. On y sent le désir de retrouver un rapport au langage proche de celui de l'enfant. On vous imagine, toute petite, écrivant déjà. Vous avez publié La mer à boire, une nouvelle, en 1980. Vous aviez alors vingt-trois ans; c'est relativement jeune pour une première publication. Pouvez-vous me parler de votre venue à l'écriture?

ÉLISE TURCOTTE J'ai commencé jeune à écrire, et sans m'en rendre compte. C'est en effet comme si j'avais toujours écrit. J'écris depuis l'âge de dix ou douze ans. Je ne me souviens pas du moment où je me suis dit que j'allais devenir écrivaine. Ça c'est passé beaucoup plus tard. J'ai toujours écrit dans des cahiers. J'aime beaucoup écrire à la main. J'aime le geste, c'est très sensuel, un peu comme se maquiller le matin. $\mathrm{Si}$ j'avais su dessiner, c'est peut-être ce que j'aurais fait. Ma venue à l'écriture, c'est un peu ça. Et puis il y a eu la lecture : j'ai commencé à écrire parce que j'ai beaucoup lu. Je lisais énormément quand j'étais jeune. C'est dans l'univers des livres que je me reconnaissais le mieux.

voix ET IMAGES Ce qui fait croire que vous écrivez depuis toujours, c'est aussi l'impression que votre rapport au réel tient de la lecture, que vous le décodez comme on déchiffrerait un manuscrit.

ÉLISE TURCoTTE Tout à fait. Je lis malgré moi, même ici, maintenant. Quand j'ai commencé à écrire de la prose, j'étais obsédée: tout ce que je voyais devenait une phrase de roman. Ça m'a donné des ailes. Une sorte d'ouverture, et d'un seul coup. En même temps est venue la tension. J'étais tendue comme un arc. C'est de cette tension qu'est faite la prose narrative.

voIX ET IMAGES Dans Caravane, c'est très frappant; les mots y apparaissent comme des personnages.

ÉLISE TURCoTTE C'était particulièrement fort quand j'écrivais Caravane. J'avais toujours un carnet sur moi. Quand je voyageais en métro, par exemple, tout y devenait un début de nouvelle, de roman. Je suis encore comme ça. C'est peut-être une façon différente de voir le réel. En période d'écriture, il y a des moments où tout ce que je dis devient une phrase, un titre potentiels. On peut donc dire en effet que je lis le réel. Comme s'il allait devenir un roman. Mais réciproquement, les romans et la poésie me parlent beaucoup du réel. 
voix Eт IMAGES Votre génération vient après celle des Nicole Brossard, France Théoret, Madeleine Gagnon, des écrivaines qui ont beaucoup travaillé à partir de l'introspection, de l'intimité, du désir, du rapport au corps, sans jamais se départir de leur conscience d'être femme. La plupart de vos livres sont écrits à la première personne. Mettez-vous une attention particulière à écrire la subjectivité féminine? La conscience d'être sexuée vous anime-t-elle lorsque vous écrivez?

ÉLISE TURCotre Je n'y pense pas, c'est naturel. C'est peut-être aussi ce qui distingue ma génération de celle qui l'a précédée. En disant cela, je ne souscris pas du tout au discours antiféministe. Je me considère féministe, je l'ai toujours été et le suis encore plus aujourd'hui, car je trouve que la situation des femmes régresse au lieu de s'améliorer. Mais quand il s'agit d'écriture, j'essaie d'être en adéquation avec ma propre vision, celle que je veux mettre sur papier. Alors je ne me pose pas ce genre de question. Je n'en ai pas besoin. Je n'essaie pas de reproduire quoi que ce soit, je veux écrire, et par l'écriture, comprendre. Et cette compréhension, ce mode de connaissance passent nécessairement par un corps sexué: celui qui écrit.

VoIX ET IMAGES Pourriez-vous écrire en vous plaçant dans un corps masculin? ÉLISE TURCotre Je ne pense pas. Je me suis déjà posé la question, mais je ne l'ai jamais fait. Je ne vois pas pourquoi je le ferais. D'ailleurs, je dois toujours retravailler mes personnages masculins. C'est une question qu'on m'a souvent posée. On m'a également fait remarquer qu'il n'y avait pas d'hommes dans mes livres, qu'ils étaient tous effacés. Et alors? Qu'est-ce que ça peut faire? J'ai un frère, mais je viens d'une famille de quatre filles. La langue maternelle, c'est celle qui nous vient de notre mère. Je suis une femme. Ce sont ces questions-là qui m'intéressent, et mon jeu est un jeu féminin; je ne peux pas le transformer. J'ai aussi une sorte, comment dire... $\mathrm{d}^{\prime}$ «inconscience» quand j'écris - mais pas au sens péjoratif. Il y a une part d'inconnu dans l'aventure de l'écriture, et j'y tiens. Je m'y accroche même. Mais d'abord, je dois franchir des barrières, faire tomber des cloisons, mettre K.-O. de vieilles amies: l'anxiété, l'angoisse... Pour moi, le temps de l'écriture échappe plus ou moins au temps social. Ce qui ne veut pas dire que l'écriture ne comporte pas une dimension politique ou sociale, mais qu'elle me place, en quelque sorte, en dehors du monde, probablement pour mieux le réintégrer après coup. Je m'absente un moment du monde, en emportant avec moi une parcelle afin de travailler à le rendre plus visible. C'est sans doute ce qui me permet d'échapper au Surmoi, et même au Moi. Sinon, je n'écrirais pas les mêmes livres. Je tends vers une écriture de plus en plus organique. Je voudrais que les mots trouvent leur forme le plus naturellement possible. Ainsi, me placer dans un corps masculin devrait être quelque chose de naturel et non de fabriqué. Difficile...

voix ET IMAGES Sans doute sommes-nous habitués de voir les sujets féminins aux prises avec leur Surmoi. Or, ce n'est pas du tout le cas de vos personnages. On peut être déconcerté par une telle liberté dans l'expression du rapport au corps, à la sexualité, à l'identité. Une liberté de la contradiction aussi. Souvent, les personnages féminins vivent mal leurs contradictions, se sentent déchirés, alors que les vôtres n'en éprouvent aucun malaise.

ÉLISE TURCotre Non, parce que c'est leur moteur. Ce sont des êtres contradictoires. C'est cela qui crée les personnages. Prenons par exemple la culpabilité: tous mes 
personnages portent une grande part de culpabilité, ça fait partie d'elles. Je ne peux pas les retenir; elles vivent avec la culpabilité comme nous-mêmes vivons avec elle - je veux dire les femmes de notre génération.

voIX ET IMAGES Il est vrai que le doute et la culpabilité sont importants dans vos écrits. On peut y voir la critique d'une certaine culture québécoise, notamment l'héritage religieux. Mais on sent aussi une volonté non tant de s'affranchir de la culpabilité et du doute que d'en faire des ressorts de l'écriture et de l'action, une vision alternative du réel en somme, qui serait aussi une façon d'accepter notre condition.

ÉLISE TURCoTte Oui. Maintenant je suis capable de considérer cela avec du recul. Et je réalise que tous mes personnages sont sur la corde raide, toujours à la frontière d'un changement de vie, entre deux choses. Ça leur donne une sorte de liberté, malgré tout, malgré leur nature, et même malgré moi. Quand on ne sait pas où on est, une liberté de pensée s'installe.

voIX ET IMAGES Est-ce ce qui vous incite à faire de la rupture un thème privilégié? ÉLISE TURCOTTE Oui. La rupture est un moment de transition où il peut se passer beaucoup de choses, et en cela comporte une dimension très positive. Il y a toujours quelque chose qui naît d'une rupture. Sans doute l'écriture, dans mon cas. Mais surtout, ça place mes personnages en état de déséquilibre, et c'est précisément ce que je recherche. Même si je décrivais un couple qui fonctionne, il se trouverait un déséquilibre quelque part. Tous mes personnages perçoivent l'amour comme relevant du tout ou rien. C'était aussi mon cas, étant plus jeune. Je me reconnaissais dans les personnages de Réjean Ducharme : il n'y avait jamais assez d'amour, on n'en recevait jamais assez, on n'en donnait jamais assez. Comme si la soif d'amour ne pouvait jamais être comblée. Le don jamais assez donné. Socialement, la rupture est aussi quelque chose d'important. Ça relève de l'échec, de la désorganisation, de la perte d'illusion. Il y avait tout, et voilà qu'il n'y a plus rien. Et puis l'amour, les rapports hommes/ femmes, ça demeure un thème primordial en littérature.

VoIX ET IMAGES La rupture permet en outre une double vision, orientée à la fois vers le passé et vers l'avenir, et qu'on n'a pas toujours: quand on est dans l'action, on regarde vers l'avant.

ÉLISE TURCOTTE Une double vision, oui. Une vision noire aussi, enfin assez noire, car il y a beaucoup d'humour dans certains de mes livres. Mes deux derniers livres ne sont pas drôles du tout, mais dans Caravane, il y a de l'humour, de l'autodérision aussi, qui est une autre forme de la vision noire.

VoIX ET IMAGES Je ne sais pas si on peut parler d'humour, mais il y a en tout cas des phrases étonnantes, où se juxtaposent des éléments hétéroclites, ce qui rend votre écriture si caractéristique. Vous ne travaillez pas dans la linéarité, ni narrative ni temporelle. Votre syntaxe opère constamment des courts-circuits. Dans votre poésie en prose, c'est assez fulgurant. Il arrive aussi que les phrases n'aient apparemment aucun rapport entre elles. Cette architecture crée des ouvertures sémantiques, des fissures qui à la fois creusent le sens et l'élèvent.

ÉLISE TURCoTte La fissure, le vertige... c'est cela. Je travaille par strates, à la verticale. C'est d'ailleurs ainsi que je lis le réel. Ça me vient naturellement, même en parlant: je fais des conjonctions entre des phrases qui ne devraient pas aller 
ensemble. Mais comme mes phrases ne sont pas alambiquées, on y voit une écriture limpide, transparente, tranquille. C'est peut-être que les gens sont incapables d'analyser les syntagmes les uns à la suite des autres; il y a une syntaxe là-dedans qui... semble échapper à plusieurs. On parle souvent étrangement de mes livres. Du Bruit des choses vivantes, par exemple, on a dit que c'était un livre sur le bonheur. Mais je n'ai jamais écrit de livre sur le bonheur!

VoIX ET IMAGES Il y a peut-être une joie dans ce livre, ou l'apprentissage...

ÉLISE TURCOTTE Mais le mot «joie» est déjà plus subversif, il me semble, plus vertical. Ce n'est pas calme une joie, ce n'est pas tranquille! Je n'ai pas une écriture tranquille.

VOIX ET IMAGES L'une des choses qui contribuent à donner cette fausse impression de tranquillité à votre écriture est peut-être que vos personnages et leurs discours ne sont jamais dans le drame, le deuil, la perte absolus. Il y a la possibilité d'un absolu, mais on n'y est pas encore.

ÉLISE TURCoTte En effet. Dans L'̂́le de la Merci, je ne voulais pas d'une famille aux prises avec un drame comme l'inceste ou la violence. Mais il y a d'autres types de drame, qui tiennent du silence. J'aime les choses qui sont difficiles à percevoir, mais néanmoins présentes. C'est un défi d'écriture. Quand je lis, c'est ce que je recherche: sentir un personnage vivre, un drame se profiler, une tension dramatique monter, sans que rien ne soit nommé, sans qu'on sache précisément ce qui se passe. Sinon, on peut facilement tomber dans le psychologisme.

voiX ET IMAGES Ce que vous évitez. La charge de violence, le trouble psychologique des personnages sont là, mais sont ressentis à travers les objets.

ÉLISE TURCotTE C'est que je travaille beaucoup, en marge du texte, sur mes personnages. J'y pense, j'écris sur eux, des choses que je ne mettrai pas dans mon livre. Pour La maison étrangère, j'ai fait beaucoup de recherches sur le Moyen Âge, pour m'imprégner de cet univers, et l'imprimer dans mon livre.

voIX ET IMAGES On le sent, sans pour autant que ce soit didactique ni que vous forciez la note.

ÉLISE TURCOTTE C'est un autre poumon, ça alimente mon écriture. Tout le travail sur l'analogie, par exemple, et tout ce qui touche au bestiaire, pour construire l'univers d'Élisabeth, il fallait que je m'en imprègne.

VoIX ET IMAGES Faites-vous toujours de la recherche avant d'écrire un roman?

ÉLISE TURCotTE Non. Généralement je n'en fais pas. En fait, j'en ai fait un peu pour L'̂̂le de la Merci, même si je ne voulais pas écrire sur les adolescents. J'ai fait quelques appels, j'ai lu sur le suicide. Mais ce n'est pas ce que j'appellerais de la recherche. C'est plutôt une nourriture, comment dire... langagière, pour me donner un langage. Mon but n'était pas de reproduire le réel. Je voulais simplement savoir s'il était vraisemblable de se suicider de cette façon, et à cet âge. J'ai téléphoné chez Action suicide... et je me suis fait engueuler! On m'a dit: «Vous ne pouvez pas écrire ça dans un livre, vous ne pouvez pas faire un personnage de suicidé, etc.» J'ai raccroché. J'étais bouleversée. Je me sentais affreusement coupable.

voIX ET IMAGES Est-ce que ça vous a empêchée de poursuivre?

ÉLISE TURCoTte Ça ne m'a pas empêchée du tout, parce que j'ai la tête dure, mais j'étais mal, tout au long de l'écriture de ce roman. 
voiX ET IMAGES C'est un livre à part que L'île de la Merci, très différent de vos autres livres. Les personnages y sont uniques. Même le style est différent; l'écriture y est plus factuelle, plus sobre.

ÉLISE TURCoTTE Il est beaucoup moins poétique que les autres. Hélène ne me ressemble pas du tout. J'étais très différente d'elle à son âge. C'est un personnage que j'aime beaucoup, mais qui n'est pas particulièrement sympathique. Et puis elle vit dans un univers tellement fermé, j'étouffais en écrivant. Mais c'est aussi en tant que mère que je me sentais mal d'écrire ce livre. Je me disais: "Voilà un livre que je ne veux pas que mes enfants lisent.»

voIX ET IMAGES Pourtant, vos enfants étaient adolescents au moment où vous l'avez écrit.

ÉLISE TURCoTte Oui, et je voulais d'autant moins qu'ils sachent que j'avais ça dans la tête. C'est quand même moi qui l'ai écrit, même si ce n'est pas ma vie. Alors, s'il y a un livre que je ne veux pas qu'ils lisent, c'est celui-là. C'est étrange, cette crainte. Et en même temps il faut continuer. C'est aussi ça, être écrivain.

voix ex IMAGEs Dans vos livres, le tout ou rien, la précarité, la fragilité, sont des façons d'être, des modes d'apparaître. Cela est renforcé par l'abondance des phrases antagoniques et des oxymores. Je vous donne deux citations au hasard; j'aurais pu les multiplier: "Tout commence et tout est terminé», dans La terre est ici; " [t] out commence par le chant quand là tout finit», dans La voix de Carla. Outre le fait que vos personnages assument leurs contradictions, ne serait-ce pas là pour eux la seule vérité envisageable, une alternative à la connaissance scientifique ou à la conscience rationnelle?

ÉLISE TURCOTTE Je ne vois là aucune contradiction. Il y a un moment où on veut tout vivre, comme Élisabeth, enfant, quand elle observe un tableau au musée, qu'elle est incapable de l'avaler et qu'elle se sent très mal, parce qu'elle voudrait que le tableau soit en elle. Ça, c'est le tout. Puis, à la fin du livre, elle se rend compte que le rien aussi est important. Être rien fait qu'on est. Ce sont deux chemins différents. Deux façons de toucher à la vie. Soit le tout: tout vivre, tout prendre, tout gober, être dans l'extrême; soit le rien du tout: observer, être un réceptacle de la vie, comme Élisabeth le décrit. Ce n'est pas contradictoire pour moi. Même dans ma vie, je suis souvent entre les deux.

VoIX ET IMAGES Les lieux familiers sont très importants dans vos livres. La maison, la chambre revêtent un caractère de nécessité; elles ont pour fonction de protéger le monde et le sujet du chaos et de la dissémination. En même temps, on a l'impression, notamment dans Le bruit des choses vivantes et Sombre ménagerie, que l'écriture se fait depuis ces lieux mêmes, c'est-à-dire qu'il y a à la fois une distance par rapport à ces lieux (ils sont décrits) et une proximité (on s'y trouve). La maison, c'est aussi le corps, dans La maison étrangère. Comment vivez-vous votre propre rapport aux lieux quand vous écrivez? Sentez-vous le besoin d'être dans un endroit en particulier?

ÉLISE TURCotTE Oui. J'écris dans ma maison, dans mon bureau. Jamais dans un café. Bien sûr, j'ai des carnets, je prends des notes - d'ailleurs, je prends plus de notes qu'avant - , mais j'écris chez moi et même si j'ai une grande maison, j'ai beaucoup de difficulté à y écrire quand il y a quelqu'un d'autre que moi. C'est pourquoi je passe de longs moments sans écrire. Je suis très solitaire, et même sauvage. Je rêve 
d'une cabane dans le bois. Je suis obsédée par la figure de l'ermite, l'idée d'être poussée à bout, dans sa propre culture, par la solitude. C'est ce que je voulais dire en affirmant que l'écriture échappe au temps social.

VOIX ET IMAGES La maison, c'est vraiment une protection?

ÉLISE TURCotTE Oui. En même temps, comme Pierre Nepveu l'a remarqué dans Lectures des lieux, c'est un lieu où tout bouge, où tout se déplace, qui protège du chaos, mais aussi d'où le chaos peut partir. Si on a besoin de se protéger, c'est que le chaos est également à l'intérieur de soi. On m'a fait remarquer qu'il y avait toujours, dans mes livres, une dichotomie entre l'extérieur et l'intérieur. C'est présent malgré moi, comme une chose à laquelle j'assiste. Dans Caravane, le personnage est derrière la fenêtre. On a l'impression qu'elle est témoin de ce qui se passe à l'extérieur. Je me sens souvent voyeuse de ce qui se passe à l'extérieur de ma maison, dans le métro, dans l'autobus; je suis très curieuse.

voIX ET IMAGES Dans Le bruit des choses vivantes, Maria imagine sa mère en train de l'observer à son insu alors qu'elle fait de la gymnastique. Ça donne non seulement une valeur à l'activité, mais une identité à l'enfant, et un sentiment de bien-être et de sécurité, cette idée d'être observée.

ÉLISE TURCOTTE Il y a quelque chose d'étrange dans le fait d'être regardée à son insu... et de l'imaginer, parce qu'enfin c'est de l'écrire qui est intéressant, pas de constater que ça arrive. C'est comme se percevoir autrement, se percevoir autre. L'écriture est une sorte de jeu de miroir.

VoIX ET IMAGES Vos personnages se décrivent souvent comme s'ils s'observaient de l'extérieur, par des formules du genre: «je suis une fille qui fait...»; «je suis un personnage», etc. Est-ce à dire que chaque personnage possède plusieurs identités, distanciées, impossibles à englober, et que cette multiplicité et cette distanciation seraient la seule façon d'accéder à l'identité?

ÉLISE TURCOTTE Ou de la perdre, parce que, comme je l'ai dit, mes personnages sont souvent à un moment où leur vie est sur le point de basculer, et alors l'idée serait de perdre son identité, pas pour en prendre une autre, mais pour s'en défaire comme d'un vieux manteau.

VoIX ET IMAGES Comme la peau d'un serpent? L'image revient souvent.

ÉLISE TURCoTte Oui. Dans La maison étrangère, c'est ce qu'Élisabeth tente de faire. Elle dit même vouloir prendre l'identité d'une roche, d'un arbre, n'être rien... Elle a un rapport particulier à la nature, aux animaux. Il y a beaucoup d'animaux dans mes livres maintenant, alors qu'il n'y en avait pas auparavant. Je n'ai pas encore compris d'où ça venait. C'est peut-être une nouvelle façon d'approcher le monde de l'enfance. Je n'ai jamais idéalisé le monde de l'enfance; au contraire. Je ne crois pas que les enfants soient aussi purs et candides qu'on le croit. Leur vision du monde peut être très dérangeante, parfois. Mais leur monde est le même que le nôtre. Il n'y aurait que celui des animaux, au fond, qui soit différent, complètement parallèle au nôtre. Ëlisabeth croit que les animaux ont une âme, alors que son ex-conjoint dit qu'ils n'en ont pas. Elle finit par comprendre qu'ils n'en ont pas, parce qu'ils n'en ont pas besoin: eux, ils sont, ils savent être. C'est l'être pur. Peut-être cela est-il lié au fait que mes personnages se décrivent volontairement dans la distance, comme des êtres en perte d'identité. 
VoIX ET IMAGES Ce serait alors la reconnaissance de l'altérité constitutive de l'identité que permet le contact avec les animaux. Cette distance me fait aussi penser à la temporalité dans vos récits. Il y a toujours un avant et un après aux histoires que vous racontez, mais le après, on ne le connaît pas, on ne le connaît jamais. On s'imagine que la poursuite de l'histoire, c'est le fil retrouvé, mais peut-être ce fil ne se retrouve-t-il pas? Peut-être la révélation ultime est-elle cette prise de conscience que le fil n'existe pas, qu'on est constitué d'une multitude, qu'en somme l'identité n'existe pas...

ÉLISE TURCotTE En effet. D'ailleurs, ce qu'Élisabeth tente de faire, c'est de trouver le moment où l'on sait que l'on vit. Clarisse Lispector dit en substance que vivre n'est rien, c'est savoir que l'on vit qui est tout. Or, savoir que l'on vit, ça veut simplement dire être comme une pierre, comme un chat ou... s'arrêter. Être. Être au-delà ou en deçà de l'identité qu'on nous a donnée. J'ai dit que je ne pensais jamais à la subjectivité féminine, mais dans le cas d'Élisabeth, j'y ai pensé, parce que c'est une femme dans la quarantaine, avec des couches d'identité, comme on en a toutes. Il y a des couches de notre identité qui ne nous appartiennent pas, qui nous sont échues parce que nous sommes des femmes d'une génération donnée, par exemple, et dont Élisabeth veut se défaire. Ça débouche sur la question du vieillissement du corps, de notre non-place dans une société qui glorifie la jeunesse, la perfection, l'excellence. J'ai voulu prendre le contre-pied de cette réalité. Ce qui m'intéressait, c'était l'échec, le moment où elle décide de renoncer à cette part de son identité qui n'est pas vraiment elle, qui lui est imposée. Les instruments de sa transformation, ce sont la noirceur, la traversée de la forêt, l'échec, le renoncement. C'est très marqué dans ce cas-ci, mais je pense que c'est aussi présent dans mes autres livres.

voIX ET IMAGES On l'observe chez Albanie, à travers les variations de la lumière, le passage des saisons. Tout est observé de façon minutieuse, et c'est ce qui marque les modulations identitaires du personnage en transformation. Dans Caravane, c'est davantage lié à des événements, parce que le personnage vieillit.

ÉLISE TURCotTE Le personnage de Caravane, je l'ai sorti de la maison. Presque tous les lieux où elle se trouve sont extérieurs. Il y a toujours une menace dans mes livres, même dans ma poésie. Le monde est menaçant. Ça installe une tension sous-jacente. La tension doit toujours apparaître sous et non pas dans l'écriture. Aujourd'hui, la mode est à l'écriture "coup de poing", pour reprendre une expression de journaliste - c'est un terme que je déteste. Ce qui a du succès c'est une "écriture coup de poing", des "phrases coup de poing", une "pièce de théâtre coup de poing".

VoIX ET IMAGES Sans qu'on sache précisément ce que ça veut dire...

ÉLISE TURCotTE Ça ne veut rien dire. Ou ça veut dire que ce qui frappe, c'est ce qui frappe immédiatement. Moi j'aime beaucoup plus l'uppercut que le jab, pour poursuivre dans des termes de boxe. Ce n'est pas parce que l'écriture semble tranquille qu'elle ne nous donne pas un coup de poing par en dessous... J'aime que la tension soit sous les mots, qu'on ne sache pas où elle est, comme chez Raymond Carver, par exemple. Plusieurs écritures de femmes sont ainsi: leur force vient d'une fausse simplicité, et toute la tension, la complexité sémantique sont sous le texte.

voIX ET IMAGES Doit-on voir là une forme de retenue? Parce qu'il me semble y avoir une retenue dans votre refus de finir certaines phrases. Et ces interruptions 
contribuent elles aussi à ouvrir le sens. C'est souvent de là qu'arrive l'uppercut, du non-dit, et le sens alors surgit comme un geyser...

ÉLISE TURCoTte Oui. C'est la fissure dont vous parliez plus tôt - même si je n'aime pas non plus ce mot. Le non-dit se situe souvent entre deux phrases, deux thèmes hétérogènes. C'est ça qui dit tout.

VoIX ET IMAGES Cela exige du travail, une ascèse, mais aussi une confiance dans l'acte de lecture. Vous me dites être étonnée par la lecture qu'on fait de vos livres. Mais vous ne croyez pas non plus en la facilité. L'esthétique coup de poing participe d'une certaine facilité; pas celle que vous pratiquez.

ÉLISE TURCotTE Mais je n'écris pas pour les lecteurs, je n'écris pour personne... enfin j'écris peut-être pour quelqu'un, mais je ne pense pas aux lecteurs quand j'écris. Je ne crois pas qu'il faille y penser non plus. Cela dit, je fais confiance aux lecteurs, à ce que chacun trouve dans mes livres.

voıX ET IMAGES J'ai évoqué l'impression que vous lisiez le réel, qu'il se présentait à vous comme un code à déchiffrer. Ça semble être tout un programme. Ça donne à vos livres leur texture particulière, leur profondeur de champ, si je peux dire, en même temps que ça leur fournit leur matière. Cela vous suffit-il ? Vous abandonnez-vous à l'errance de vos personnages ou avez-vous besoin d'un plan pour travailler?

ÉLISE TURCotTE C'est curieux que vous disiez cela. Il se trouve que j'ai commencé à réfléchir au thème de l'errance il y a une semaine ou deux, en réalisant que ça faisait partie de tous mes personnages. C'est quelque chose que je souhaite explorer plus avant. Mais pour répondre à votre question: oui, je m'adonne à l'errance. Les plans, je les fais à mesure. En fait, ce ne sont pas des plans, du moins pas des plans linéaires; ce sont des plans verticaux. J'écris à l'ordinateur, mais j'ai de nombreux cahiers de notes, et j'établis des correspondances, des liens entre tel symbole, tel personnage, etc. Tout cela est nourri par des objets qui passent dans mon bureau, beaucoup de réflexions, de lectures aussi, pendant que j'écris. Aussi ai-je un peu l'impression de faire une «tapisserie » - j'emploie ce mot en pensant à La maison étrangère - , avec sa texture, son architecture particulière, où tous les motifs sont enchevêtrés.

VoIX ET IMAGES Est-ce que ce sont vos cahiers de notes qui se retrouvent dans vos romans? Parce qu'il y a toujours des cahiers. Albanie travaille avec sa fille dans un cahier, à écrire des rêves, à consigner le quotidien. Élisabeth aussi a un cahier, où elle écrit ses pensées. Dans L'île de la merci, Hélène a un scrap-book, où elle rassemble des coupures de journaux. Ce sont un peu comme des livres dans le livre.

ÉLISE TURCOTTE Je n'avais pas remarqué que tous mes personnages avaient des cahiers. Peut-être est-ce une façon pour elles de collectionner le réel, des éléments du réel? J'ai souvent fait cette supposition: s'il y avait une fin du monde, et si quelqu'un par la suite essayait de découvrir ce qu'il y a eu sur terre, il aurait ces cahiers. Quelqu'un arrive sur la terre et trouve un cahier avec des photos de meurtriers, des articles sur des meurtres, ou encore un carnet avec des photographies d'animaux. C'est ainsi que je les imagine.

voIX ex IMAGES Dans Le bruit des choses vivantes, c'est clairement évoqué. Albanie dit que si jamais elle disparaissait, le cahier aurait valeur de passé pour sa fille. C'est là une façon de contrer la menace de la perte, de faire en sorte que la mémoire ne soit pas effacée dans l'éventualité de la mort, de créer un artefact... 
ÉLISE TURCOTTE Oui, mais à la manière d'un collectionneur. C'est une sorte d'hyperréalisme. Par exemple, j'aime beaucoup les listes, toutes sortes de listes. Parfois je me dis que ce serait agréable de faire un livre juste avec des listes, des listes d'épicerie, de choses à faire, etc. C'est comme un besoin obsessif de ramasser, de collectionner des objets. En écrivant La maison étrangère, j'étais habitée par l'idée de décrire un monde en train de disparaître. J'avais l'impression de construire un navire, une sorte d'arche de Noé, en imaginant que tel objet, telle chose désirée seraient tout ce qui resterait de notre monde. C'est là un désir d'être témoin, de dire voilà : il y a eu ceci, cela, on a vécu là. Il en va de même dans mes poèmes. Dans La terre est ici, par exemple, que j'ai imaginé comme une collection de portraits : images, pierres, coquillages, paysages, chiffres et mots dévoilant un aspect de la vie sur terre.

VoIX ET IMAGES Le témoignage est un peu comme ce regard posé sur l'autre sans qu'il le sache, et qui lui donne plus de réalité.

ÉLISE TURCOTTE Oui. J'aime aussi beaucoup les cimetières, les inscriptions sur les stèles : lire les dates et les noms me fait un effet... probablement romanesque, je ne sais pas. Je ne suis pas fascinée par mon propre passage sur la terre, mais par celui des autres.

voIX ET IMAGES D'où vous vient cette fascination pour la mort? Elle est de plus en plus présente, en particulier dans votre poésie.

ÉLISE TURCOTTE La mort est une réalité que je dois apprivoiser. Mon nouveau livre s'intitule Archiviste de la mort et porte entièrement sur la mort. J'y suis dans l'errance totale. À chaque nouveau livre, j'aime faire quelque chose que je n'ai pas fait avant. Je n'aime pas marcher dans mes propres traces. Je ne sais même pas quel est le genre de ce livre; ce n'est pas un roman, ce ne sont pas des nouvelles... je n'ai encore aucun repère. Le premier texte s'intitule «La mort et moi » $j^{\prime}$ y explique que j'ai toujours vécu en compagnie de la mort; tous les jours j'y pense, je ne sais pas pourquoi. Déjà, quand j'étais jeune, ça m'habitait, à cause de questions existentielles du genre: «Ã quoi ça sert de vivre si on va mourir? ? Mais quand on a des enfants, ça nous hante. Dans Le bruit des choses vivantes, la question est clairement posée: comment vivre avec l'idée et surmonter la possibilité de la perte? Récemment, cette préoccupation a pris une dimension physique: le corps mort et toutes les questions qui s'y rattachent m'intéressent. Je me documente sur les rituels entourant la mort à travers le monde. Je suis très préoccupée par la façon dont on traite les morts aujourd'hui. On est totalement dans la dénégation. Quand mon père est mort l'an dernier, j'ai eu la sensation que sa mort m'avait été volée, que je n'avais pas pu bien la vivre... même si on peut difficilement «bien vivre» la mort de quelqu'un. J'étais présente au moment du décès. Mais après la morgue tout va tellement vite: le corps part si vite, on l'emmène et vous ne savez plus quoi faire. Avant, on veillait les morts, on leur laissait, si je peux dire, le temps de s'en aller. Certains peuples croient que ça prend tant d'heures à l'âme pour quitter le corps, et qu'il faut rester à ses côtés. Qu'on y croie ou pas, il me semble y avoir là quelque chose d'important. Même d'un point de vue médical, il y a un certain temps durant lequel des fonctions vitales continuent. On devrait, me semble-t-il, veiller le corps tant que ces fonctions ne sont pas éteintes. Mais on ne le fait pas. Pourquoi? On a peur de la mort, elle représente quelque chose de laid, de vieux, de sale. Peut-être cette fascination est-elle liée à la 
présence grandissante des animaux dans mes livres, eux qui n'ont pas du tout le même rapport à la mort que nous?

VoIX ET IMAGES Sombre ménagerie et La maison étrangère ont paru la même année. En lisant le récit de rêve où il est question d'oiseaux, à la page 136 de La maison étrangère, je me suis dit: "voilà la "sombre ménagerie"»! Évidemment, les oiseaux reviennent, dans Sombre ménagerie. Il y a aussi les poèmes, écrits en vers très courts, et présentés en forme de chute, ce qui est plutôt rare chez vous, et qui contraste d'autant avec la prose. Ce mouvement de chute, très sensible, m'amène à penser que les animaux seraient peut-être une façon d'apprivoiser la mort, de l'approcher.

ÉLISE TURCoTte Peut-être en tout cas de la regarder telle qu'elle est. Ce serait déjà ça. Mais cette fascination pour la mort a toujours été présente chez moi, car je suis une grande lectrice de romans policiers.

VoIX ET IMAGES On le devine en lisant Lîle de la Merci.

ÉLISE TURCoTte J'aurais aimé être détective. J'aime toutes les histoires d'enquête, de meurtre, de procès, d'avocats. Ma fascination pour la mort en est aussi une pour la violence de la mort, et ses causes. Le travail d'enquête que fait le détective dans un roman policier, c'est un peu ce qu'on fait quand on écrit. Ce n'est pas un hasard si plusieurs écrivains adorent le roman policier. Le personnage du détective ressemble à celui de l'écrivain; les deux sont à la recherche d'indices, s'attachent à construire une espèce de cosmogonie d'objets qui nous disent: ici, l'être humain est passé.

VoIX ET IMAGES Vos personnages semblent constamment en attente de signes, ce qui correspond certainement à une acuité du regard. Ils sont en attente d'une coïncidence d'où surgira le sens. D'ailleurs, Albanie l'affirme: la vie est une coïncidence.

ÉLISE TURCOTTE Les emblèmes, les objets, les signes : tout cela est en jeu. Albanie dit, par exemple: «Une chemise mal attachée, c'est le signe que l'univers va s'écrouler aujourd'hui.» Tous mes personnages sont ainsi. Élisabeth met une robe bleue pour telle occasion. Même dans mes poèmes, il y a des couleurs de rouge à lèvres pour telle ou telle circonstance. C'est comme une façon de conjurer la mort, tout en se disant «je suis mortelle, je suis complètement mortelle».

voix ET IMAGES C'est aussi une façon de retrouver le fil, le fameux fil de notre identité disloquée, et qui n'est peut-être rien d'autre que le lien qui unit les objets. N'est-ce pas là l'une des fonctions des objets?

ÉLISE TURCotre Les objets sont reliés entre eux par le fil du désir, de la volonté de savoir que nous avons vécu, et aimé.

voIX ET IMAGES D'ailleurs ils ne sont presque jamais présentés individuellement. On ne parle pas du téléphone, par exemple, ou de la radio; on invoque plutôt «les choses» ou «les objets». Ça donne l'impression d'un amas, d'une sorte de nébuleuse, à quoi le sujet se heurte.

ÉLISE TURCOTTE Il est vrai que les objets forment une constellation, ou une nébuleuse, dans le monde de mes personnages. Quand je crée un personnage, il y a toujours des objets qui l'entourent, le définissent. Ce sont autant de motifs sur la partition du personnage, de la même façon que chez Milan Kundera, par exemple, le chapeau melon est un motif; dans L'insoutenable légèreté de l'être, il est le fil qui relie Thomas à Sabina, il revient en rêve, etc. Pour moi, les objets sont des emblèmes. 
Voix ET IMAGES Vous entourez-vous des objets de vos personnages quand vous écrivez?

ÉLISE TURCotTE Pas nécessairement ceux de mes personnages. J'aime beaucoup les objets, mais en même temps j'ai un rapport conflictuel avec eux. Parfois j'ai l'impression qu'ils sont contre moi, qu'ils m'en veulent. Lorsqu'ils sèment le chaos dans la maison.

VoIX ET IMAGES Le chaos se manifeste en effet à travers le désordre. Dans Lîle de la Merci, quand Hélène fait du rangement, c'est pour elle une façon de combattre le chaos: quand sa chambre est en ordre, que chaque chose est à sa place, elle se sent en paix, en sécurité.

ÉLISE TURCotTE C'est d'ailleurs le seul personnage aussi ordonné, aussi peu dans l'errance.

voix ET IMAGES Mais elle est tout de même en quête, en errance intérieure. Elle se cherche.

ÉLISE TURCotrte Oui. Sa vie est en train de basculer. En fait elle a déjà basculé. C'est peut-être le seul de mes romans où la vie bascule vraiment. Le seul où «la catastrophe» annoncée, pour reprendre un de mes titres, non seulement est imminente, mais arrive. C'est d'ailleurs le seul que j'ai écrit en connaissant la fin.

Voix ET IMAGES Les situations se retournent souvent dans vos textes, autant dans votre poésie que dans vos romans. Dans L'île de la Merci, peut-être le drame est-il vécu extérieurement, et alors ce serait sa résonance que vit intérieurement Hélène?

ÉLISE TURCotTE La menace, je l'ai dit, vient souvent du monde extérieur. Mais dans L'̂̂le de la Merci, elle vient aussi de la maison. Alors que généralement la maison protège - de la violence, du violeur, du meurtrier - , ici, elle menace par son silence. La menace vient à la fois du cri à l'extérieur et du silence à l'intérieur. Là où tout dérape, c'est quand les deux s'entrechoquent dans l'esprit d'Hélène. D'ailleurs, il n'y a pas de véritable dichotomie entre l'extérieur et l'intérieur, du moins pas dans ce roman. J'ai construit mon histoire en comparant la famille à un échiquier; chaque pion a une fonction. Si on déplace un pion sans respecter les règles du jeu, ça détruit le jeu. La famille d'Hélène, comme toute famille, est un échiquier : chaque membre a une image, et s'il déroge à cette image que les autres se font de lui, le déséquilibre s'installe. Le fait qu'Hélène décide de sortir de la maison pour aller travailler au garage déstabilise toute la maison, car c'est elle qui portait le poids de l'incapacité de ses parents.

Voix ET IMAGES Doris Lessing écrit que "c'est souvent le membre le plus "normal" d'une famille ou d'un groupe qui est malade ; mais comme ils ont de fortes personnalités, ils survivent parce que les autres, aux personnalités plus faibles, vivent leur maladie pour eux».

ÉLISE TURCotTe C'est juste. C'est pourquoi ce n'est pas Hélène qui se suicide, mais Lisa, qui, elle, n'est pas du tout névrosée. C'est le seul de mes livres où le monde intérieur et le monde extérieur entrent en collision, et c'est ce qui cause la catastrophe. Hélène a quinze ans, l'âge de la fille qui se fait violer. C'est obsédant de mettre au monde des filles lorsqu'on vit dans un milieu où ça peut arriver.

Voix ET IMAGES Dans La maison étrangère, votre protagoniste n'a pas d'enfant. Le roman nous met en présence d'une femme dans la quarantaine sans enfant, et d'une 
autre qui en a deux, mais qui chavire. Lorraine est extrêmement perturbée par la situation de ses enfants, au point de fantasmer leur meurtre, plutôt que de les laisser vivre dans ce monde horriblement dangereux. Comment faites-vous l'interface entre votre vie et votre écriture? Le fait d'avoir des enfants influence-t-il votre travail?

ÉLISE TURCotTe Oui. Avoir un premier enfant m'a fait réfléchir au temps. C'est un thème important dans Le bruit des choses vivantes, le temps qui passe. C'est aussi important quand on a des enfants... Ça m'a beaucoup rapprochée de la forme narrative. Quand on écrit de la prose, qu'elle soit linéaire ou non, il faut sentir le temps qui passe. Il y a ça, d'une part, le fait que ça influence la vie de toutes les manières possibles, même des détails quotidiens, qu'on ne peut plus décrire de la même façon. Et puis le temps en soi nous manque. C'est difficile d'observer une assiduité quand les enfants sont jeunes. C'est plus facile d'écrire des nouvelles, par exemple. Enfin, les enfants nous font voir la vie sous d'autres angles, qu'on ignore quand on n'en a pas. Je n'aurais pas écrit Le bruit des choses vivantes sans enfant, ni Caravane. Les familles défaites me stimulent beaucoup comme sujet d'écriture. Dans La maison étrangère, je voulais un personnage sans enfant, parce que je la voulais totalement solitaire. C'est un peu une façon d'explorer ce qu'aurait pu être ma vie sans enfant. Et puis il y a Lorraine. Dans tous mes livres, il y a un personnage névrotique, qui incarne la menace, qui l'a, pour ainsi dire, intégrée. Dans La maison étrangère, c'est Lorraine. Dans Le bruit des choses vivantes, c'est Agnès. Elle a perdu son frère, mais elle est beaucoup moins angoissée que Lorraine, qui vit une situation extrême. Elle souffre d'angoisse chronique et doit prendre des médicaments. C'est comme si j'avais toujours besoin, même dans un univers apparemment serein, comme celui du Bruit des choses vivantes, d'un personnage qui assume la folie du monde dans lequel on vit, la difficulté de vivre dans ce monde, l'angoisse, la paranoïa même, que cela peut engendrer. Il m'arrive, en lisant le journal par exemple, de fermer les yeux et d'imaginer que je suis Lorraine, et alors c'est épouvantable. Cette vision-là prend place dans l'écriture. Comme l'affirme Kundera, écrire un roman, c'est travailler sur un aspect, une possibilité existentielle qu'on va pousser à l'extrême à travers un ou deux personnages. Il est donc certain que le fait d'avoir des enfants m'a influencée. Raymond Carver dit pour sa part que ce qui l'a le plus marqué dans sa vie d'écrivain, c'est d'avoir eu des enfants, parce que... ça l'a empêché d'écrire et l'a obligé d'aller travailler! Selon lui, c'est beaucoup plus fort qu'une influence littéraire; c'est de l'ordre de l'influence de la lune sur les marées. Ce rapport qu'on a avec les enfants, fait de responsabilité et d'anxiété perpétuelles, nous change pour toujours.

voIX ET IMAGES Cela a-t-il modifié votre rapport avec votre enfance, en vous amenant, par exemple, à lui accorder une place plus importante sur le plan littéraire?

ÉLISE TURCoTte C'était déjà là. Dans Navires de guerre, il est beaucoup question de mon enfance. J'y décris une photo de moi comme la seule chose qui resterait de mon enfance. Il y a là quelque chose comme un enlèvement. J'ai l'impression que beaucoup d'écrivains se tournent vers leur enfance en vieillissant, parce que tout est déjà là.

voıx ET IMAGES Certains motifs récurrents semblent venir de l'enfance. La fascination pour les maisons : le plaisir d'observer ce qui se passe à l'intérieur des maisons 
et de s'inventer des histoires; les frayeurs, la peur de la guerre, des extraterrestres; la fascination pour les bateaux, l'univers marin.

ÉLISE TURCotTE Oui. Ce sont des éléments qui m'appartiennent en propre. Sauf la fascination pour le monde marin; je n'ai aucune idée d'où ça vient. Probablement parce qu'un bateau, c'est un microcosme de notre société. C'est comme la vie en prison, mais sur l'eau. Ça présente un contraste étonnant: d'un côté, on est sur l'eau, dans l'errance totale et le danger; de l'autre, c'est un univers parfaitement ordonné, hiérarchisé. J'ai toujours été fascinée par l'histoire du Titanic, bien avant qu'on en fasse un film. J'adore les catastrophes marines, les naufrages, la noyade, je suis fascinée par la noyade. Je m'en suis rendu compte en écrivant Sombre ménagerie. J'étais au milieu de mon roman quand je me suis arrêtée; j'étais fatiguée, je n'avais plus l'impulsion de continuer. Alors je me suis dit: «Il faudrait que je sombre encore plus creux pour continuer mon livre.» Ma façon de le faire a été de retourner à la poésie, ce que je n'avais pas fait depuis des années. Ça m'a permis de revenir aux racines de mon écriture, de retrouver le goût d'écrire. Sombre ménagerie, c'est très sombre, c'est la mort. Or, toute la partie du milieu se passe sous l'eau. J'ai décrit la mort comme si on était dans une ville sous-marine. Quand j'ai pensé à ma mort, j'ai pensé à la noyade. J'adore l'imagerie autour d'Ophélie. Dans L'̂́le de la Merci, il y a la rivière des Prairies, qui est très sale, et d'où des noyers peuvent remonter sur le rivage. Ce sont des traces. Beaucoup d'écrivains sont morts noyés. Et puis je suis née à Sorel. Même si je suis arrivée à Montréal à deux ans, le fleuve fait partie de ma vie. J'ai toujours voulu et pensé finir mes jours aux bords du fleuve. J'ai besoin de l'eau.

voIX ET IMAGES Vous êtes aussi attirée par les pays nordiques, le Grand Nord, les maelströms, les glaciers...

ÉLISE TURCOTTE Oui, parce que ce sont des paysages extrêmes, et en même temps presque désertiques. C'est un peu comme si la fin et le commencement du monde se retrouvaient. Remarquez, je n'y suis jamais allée; je décris intuitivement, mais je n'en sais rien. Je rêve aussi d'aller dans le Grand Nord. Ça me fascine. C'est formel aussi, cette fascination pour une beauté hors de proportions.

voix ET IMAGES Vous vous êtes arrêtée au milieu de l'écriture de La maison étrangère, dites-vous, pour vous en remettre à la poésie, voulant sombrer encore davantage. La poésie représente-t-elle à vos yeux une strate plus profonde par rapport à la prose romanesque?

ÉLISE TURCotTe Non, c'était nouveau. Quand j'ai écrit Sombre ménagerie, je ne pensais pas écrire un livre, ni publier. J'étais comme en état de survie. Élisabeth aussi a ses objets de survie, le livre qu'elle va finir par noyer, entre autres. Je l'ai donc écrit sans aucune contrainte, sans lien, en ignorant qu'il s'agissait d'un livre. J'étais très bien, même si c'était très sombre. J'étais contente de retrouver la poésie. J'en ai besoin. Je pense qu'elle nourrit mes livres. Et puis c'est peut-être vrai ce que vous dites après tout: les poèmes sont les racines qui nourrissent la prose. Il fallait que je saute, que j'aille beaucoup plus loin dans la noirceur, et cela ne pouvait se faire qu'en poésie. Il y a certaines contraintes dans un roman qu'il n'y a pas en poésie. Les personnages parlent, dialoguent. Nicole Brossard disait: «Le roman, c'est la prison, alors que la poésie, c'est la liberté.» Je n'irais peut-être pas jusque-là, mais il y a du vrai 
là-dedans. La poésie, ce sont des moments sculptés, alors que l'architecture d'un roman s'étale dans le temps.

voIX ET IMAGES Il arrive souvent que les poètes qui en viennent au roman abandonnent la poésie, comme si la poésie était l'enfance de l'art...

ÉLISE TURCOTTE Ah non, pas pour moi, au contraire! Je pensais que j'avais abandonné la poésie, parce que la prose, ça demande énormément de temps. On se trouve comme en territoire occupé... par le roman. Mais finalement j'y suis revenue, et jamais plus je n'arrêterai. Il est même possible que je ne fasse plus que cela un jour. Je n'en sais rien.

voIX ET IMAGES Diriez-vous que c'est le projet qui commande le genre? Vous ne vous dites pas, par exemple, "voici, j'écris un livre de poésie»...

ÉLISE TURCotTE Non. C'est le projet. Mais il ne s'agit pas non plus de la même impulsion. Écrire de la poésie et écrire un roman, c'est complètement différent. Ça ne vient pas de la même source, du même désir. Bien qu'au fond j'écrive mes romans comme de la poésie. J'écris tout comme de la poésie, c'est-à-dire en sculptant et en donnant une importance démesurée à chaque mot dans la phrase. Mais la poésie est plus musicale, plus jubilatoire aussi, je pense. En même temps, quand j'ai écrit Sombre ménagerie, j'avais besoin d'un vocabulaire pauvre, je cherchais la pauvreté du vocabulaire. Au début, le titre devait être Pauvre éternité. Je voulais la simplicité de formule, de langage, à la limite du presque trop simple. J'avais l'impression de décrire un monde sous la langue, en dessous du langage. C'est cela, la noyade, le monde de la mort.

VoIX ET IMAGES Qu'est-ce qui vous a finalement poussée à publier?

ÉLISE TURCOTTE J'avais un début de première partie, et à partir de là j'ai construit mon livre. Quand j'ai écrit la deuxième partie, je me suis rendu compte qu'il y avait là quelque chose d'intéressant. Et alors l'écrivain en moi a pris le dessus. J'ai vu l'architecture du livre et je me suis dit: «Je vais l'envoyer à un éditeur, et on verra bien. »

voIX ET IMAGES La mémoire de l'oubli. Vous en parlez souvent. L'oubli joue-t-il un rôle dans l'écriture?

ÉLISE TURCotTE Certainement. La mémoire aussi. C'est parce qu'on se souvient et parce qu'on oublie qu'on écrit. Ça se situe entre les deux. Souvent, lorsqu'on écrit, des choses, des sensations qu'on croyait oubliées reviennent. Mais dans La maison étrangère, c'est plus que cela, c'est l'un des thèmes principaux du livre: la culpabilité face à l'oubli. Autrement dit, je n'ai pas le droit d'oublier ce monde si dur, où des gens crèvent de faim, où il y a la guerre. C'est l'oubli qui rend Élisabeth coupable.

voIX ET IMAGES Ce que vous dites de La maison étrangère est sans doute vrai, mais il me semblait que, dans la plupart de vos livres, l'oubli était en quelque sorte revendiqué. Comme une nécessité. Comme si, une fois reconnue la faillibilité de la mémoire, il s'agissait de voir dans quelle mesure on peut en tirer parti. Est-ce que ce ne serait pas l'une des fonctions des objets que d'emmagasiner des instants présents, permettant ainsi aux personnages de s'abandonner à l'oubli sans pour autant perdre la mémoire?

ÉLISE TURCoTte Oui, tout à fait. Et le souvenir nous permettrait de passer d'un objet à un autre, d'un événement à un autre. Il s'agit d'un oubli de surface, au fond, parce 
qu'il existe une mémoire profondément enracinée, et dont on ne se rend pas compte. Un oubli de surface que viennent contrer les objets. Ainsi, comme je le disais, les objets ne sont pas là pour assurer une pérennité, mais pour désigner le moment présent, pour que nous nous assurions qu'il soit bien vécu. Mieux vécu, ou plus intensément.

voix et IMAGES Comme si l'oubli était l'occasion d'une transfiguration. Dans un poème de Navires de guerre, vous écrivez: "J'attendais des soucoupes volantes lumineuses pour venir me sortir de là.» N'est-ce pas là un mauvais souvenir d'enfance transposé en figure positive, un oubli de la frayeur au profit de la dimension salvatrice de l'image?

ÉLISE TURCotTE Vous avez raison. Peut-être que la détective-écrivaine que je suis essaie de puiser dans l'oubli, d'y récupérer des objets, des indices de ce qui a été, de ce qui est. Parce qu'on oublie même le présent, vous savez. Ainsi l'oubli est peutêtre vraiment un moteur d'écriture: on oublie et on écrit pour combattre l'oubli. Le simple fait de coucher des mots sur une page constitue une preuve d'existence. Quelque chose qu'on ne peut pas oublier.

VoIX ET IMAGES Ça répond au besoin de laisser des traces. Pas pour la postérité, mais pour le présent. Comme un témoin, un signal.

ÉLISE TURCOTTE Un signal, oui, une lumière qu'on allume pour savoir où on va. Vous savez, il m'est arrivé de penser à moi comme à quelqu'un qui n'écrit plus : ça me rend claustrophobe. Comme si ne pas écrire, ce n'était pas assez. La vie nécessite un dédoublement, constamment, sinon j'étouffe. La vie serait trop restreinte si je n'écrivais pas, j'en serais incapable. En ce sens, c'est au présent que j'ai besoin de traces.

VOIX ET IMAGES Comme d'un poumon?

ÉLISE TURCotTE C'est ça. J'aime bien cette idée du poumon. Quand j'écris, j'ai des manies. À une époque, j'avais l'impression que si je laissais l'ordinateur allumé, entre mes sessions d'écriture, un petit monde continuait de respirer sans moi : le livre. Je me disais: "Je n'ai pas besoin d'être là, le poumon respire, quand je vais revenir demain, ça ira mieux.» Parfois je laisse mes carnets ouverts, en pensant à un poumon, c'est vraiment l'image qui me vient. Ça respire, et en respirant, ça emmagasine d'autres choses.

VoIX ET IMAGES La fascination pour les histoires, qu'on trouve dans vos livres, se transforme parfois en fascination pour l'Histoire. Il y a d'un côté la petite histoire, comme dans Lîle de la Merci, celle de la fille assassinée, reprise dans les journaux, mais qui était, avant le meurtre - on le suppose - , somme toute banale. Je pense aussi à l'histoire de Félix, dans Le bruit des choses vivantes, qui relève elle aussi de la petite histoire, et qu'on doit reconstituer. Et puis il y a la grande, dans La maison étrangère, celle de l'Irlande et des Irlandais, victimes de violence et d'injustice. Si on trace un parallèle entre les histoires, d'une part celles qu'on raconte, qu'on essaie de reconstituer, qui s'inscrivent comme autant d'ajours dans le tissu du texte, et d'autre part l'Histoire, dont on ne connaît pas davantage la fin, et qui serait en même temps une ouverture aux possibles, et s'il est vrai en outre que la vie est une coïncidence, comme le dit Albanie, pourrait-on voir là une façon de donner sa chance à l'Histoire? Une chance de se racheter, d'être plus humaine? 
ÉLISE TURCoTTE Peut-être, quoique personnellement je n'y croie pas. Je n'ai pas une grande confiance dans l'humanité. Mais je crois dans la vie, la force de la vie, et peutêtre que si ces petites histoires ne sont pas finies, c'est qu'en effet elles vont se poursuivre et indiquer une issue. Prenons Félix, par exemple: on a l'impression qu'il va s'en sortir. Je n'avais jamais remarqué, c'est curieux, que ces petites histoires n'étaient pas finies. Ce sont des moments de vie en marge. Mais je pense que la grande Histoire marche en parallèle avec la petite, et qu'elles se croisent parfois. Ëlisabeth le dit, d'ailleurs. Souvent, la marche du monde semble loin de nous, mais il arrive que la petite et la grande Histoire entrent en collision. 